\title{
Facilitating Data Source Movement with Time-Division Access in Content-Centric Networking
}

\author{
Olivica Priyono ${ }^{\dagger}$, In-Yeup Kong ${ }^{\dagger+}$, Won-Joo Hwang ${ }^{++}$
}

\begin{abstract}
Wireless communication offers the flexibility to the node movement at the spatial dimension more than the wire communication not only in IP architecture but also in Content-Centric Networking. Although it gives such advantage, the intra-domain movement of a node especially the data source node affects the communication to the access point node which in the end affects the acceptance ratio of the client node that requests the data packets from the data source node. In this paper, we use time-division access method to maintain the acceptance ratio of the client node as the effect of the intra-domain data source node movement in Content-Centric Networking. The simulation result shows that the acceptance ratio of the client node can be maintained using the time-division access method as long as the interval access time is less than the coherence time.
\end{abstract}

Key words: Content-Centric Networking, intra-domain data source movement, Doppler effect

\section{INTRODUCTION}

Content-Centric Networking (CCN) is a new communication paradigm where content is $\mathrm{ac}^{-}$ cessed by the name of the content instead of the IP address [1]. It proposes the benefit of mobility support for data requester easier than the IP architecture. But its content names have a problem of privacy[2], and it gives another problem when

※ Corresponding Author: Won-Joo Hwang, Address: (621-749) Department of Information and Communications Engineering, 197 Inje-ro, Gimhae-si, GyeongsangnamDo, Korea, TEL : +82-55-320-3847, FAX : +82-55-3226275, E-mail : ichwang@inje.ac.kr

Receipt date: Feb. 7, 2014 , Revision date: Mar. 7, 2014 Approval date: Mar. 17, 2014

${ }^{+}$Department of Information and Communication System, HSV-TRC, Inje University

(E-mail:kreuz_3223@yahoo.com)

${ }^{++}$Kumoh National Institute of Technology (E-mail: iykong@kumoh.ac.kr)

${ }^{+++}$Department of Information and Communication System, HSV-TRC, Inje University

* This research was supported by the MKE (The Ministry of Knowledge Economy), Korea, under Global IT Talents Program supervised by the NIPA (National IT Industry Promotion Agency)" (NIPA-2012-H09021013). the mobility happens at the data source node. We focus on the problem from mobility. Many recent researches only examine the problem which is caused by the mobility of the data source node when it moves from one domain to another domain [3-5]. While there is a possibility that the movement of the data source node even still on the same domain can cause a problem at the receiver node or the client node.

When the data source node move from one point to another point even still at the same domain in which there is no handoff happen, it triggers problem such as fading and Doppler effect [6]. Fading happen because there is signal reflection from its environment and also from the ground plane. It leads to variations in signal strength which are received by the receiver. The fading effect can be analogously described as if we throw a bunch of balls upright. The balls will fall to the floor and spread out depend on the reflection angle. The Doppler effect represents the shifts in the frequency of electromagnetic radiation which is emitted by a moving object [7]. The example of Doppler effect can be found at the racing area. The sound 


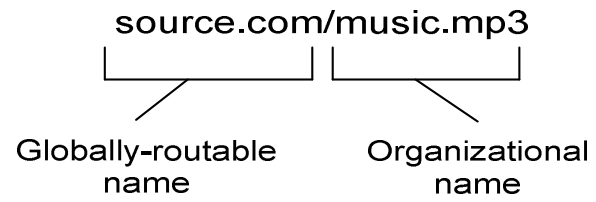

Fig. 1. Example of content name.

of the car machine gets louder when the car approaches us and softer when the car pass us by.

Thus, in this paper we proposed a time-division access method to maintain the acceptance ratio of client node as the effect of the data source node movement. Our objective is to defeat the side effect of intra-domain data source node movement with the acceptance ratio of client node as the indicator. Our contributions in this paper are:

- We show the problem that happen at the acceptance ratio of client node when the data source node is moving from one point to another point even still at the same domain (section 3)

- We design a time-division access method at the $\mathrm{CCN}$ environment to facilitating the intra- $\mathrm{do}^{-}$ main data source node movement (section 4)

- We simulate the proposed method using OPNET to verify its performance (section 5). The result shows that the time-division access method can maintain the acceptance ratio of client node as long as the interval access time is less than the coherence time.

\section{CONTENT-CENTRIC NETWORKING}

The CCN protocol contains of two primitives, namely interest and data packet. Interest packet is similar to "content query" which carries a name that identifies the desired data. Data packet is analogous to "content response" which is utilized to carry the actual content [8]. Routing in CCN can leverage aggregation through hierarchical name as shown at Figure 1. The name is like URIs with "/ character as the separating components [9]. The delimiter $(/)$ is not part of the name and not included in the packet encoding.

Every node in CCN contains of three tables, namely Content Store (CS), Pending Interest Table (PIT), and Forwarding Information Base (FIB). CS is a place to cache the data packet which passes through the node. PIT records the outstanding forwarded requests (interest packets). FIB is used to forward interest packet toward potential source(s). The forwarding model in $\mathrm{CCN}$ is described in Figure 2 . When there is a request for a data packet

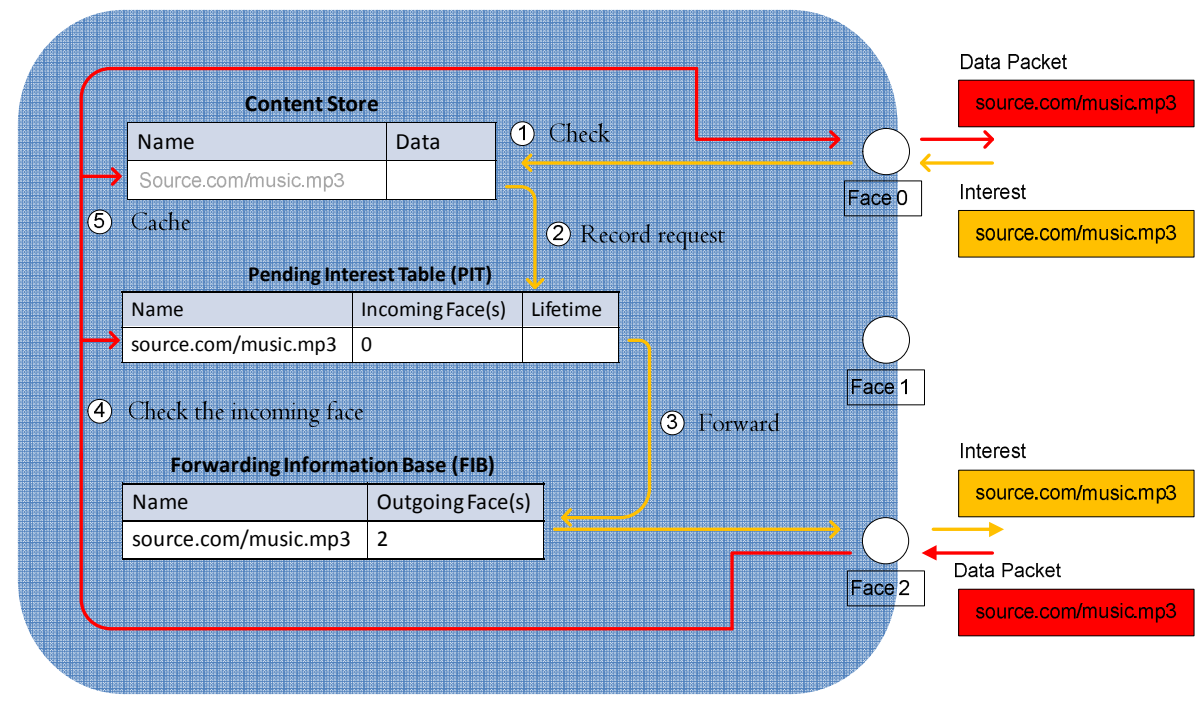

Fig. 2. Node forwarding model in $\mathrm{CCN}$. 
(interest packet), the node checks at the CS (1). If the node has the data packet, the node sends out the data packet to the incoming face of the request. If the node does not have the data packet, the node records the request in PIT by saving the incoming face of the request (2) and forwards the request based on FIB (3). When the node receives the data packet for the interest, the node checks if there is any interest have been received which ask for that data packet. If exist, the data packet is sent out the data packet to the incoming face of the interest packet (4) and caches the data packet in CS (5).

The explanation above shows that $\mathrm{CCN}$ is a $\mathrm{re}^{-}$ ceiver-driven architecture where the data packet will be produced/sent when there is a request [10]. Only interest packet is forwarded using routing mechanism. The data packet will follow the reverse-path of the interest packet. $\mathrm{CCN}$ also supports on-path caching where the requests for the same object can be answered from the caching node directly.

\section{PROBLEM STATEMENT}

The Impact of node movement for package acceptance ratio of client node is shown in [11]. Simulated using OPNET, The examination gives result which shows that the acceptance ratio of client node is decrease as the velocity increase. Figure 3 shows the connection between the data source node movement to the acceptance ratio of client node. As shown in the picture, the data source node moves with velocity from point (i) to point which has distance 1 . The distance between the access point node and the data source path is shown by $d$. The acceptance ratio is the comparison of the number of data packet received by the client node with the number of interest packet sent by the client node. At the first half of $l$, when the data source node moves closer to the access point node, the acceptance ratio of client node is $100 \%$. However, the acceptance ratio of client node de-

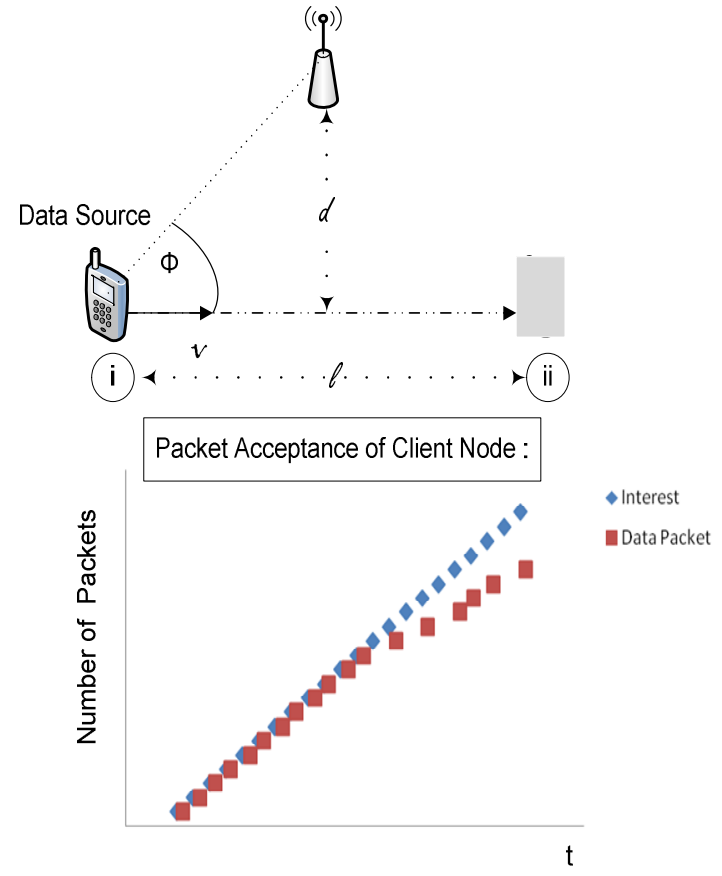

Fig. 3. The impact of data source movement to client node acceptance ratio.

creases at the rest half of 1 when the data source node moves away from the access point node. Even though the client node still constantly sends the interest packet at the same rate, the data packet received rate is decrease as the data source node moves away from the access point.

The Doppler effect as the result of node movement is commonly found in wireless communication especially in satellite systems [12]. It occurs at electromagnetic radiation emitted objects as they move towards or away from an observer. The frequency distortion under Doppler effect as a result from the movement of a mobile node can be described as:

$$
f=f_{c}+d_{f}
$$

Where $f_{c}$ is the frequency carrier and $f_{d}$ is the Doppler frequency (Doppler shift). In [13] and [14], the Doppler frequency is given by:

$$
f_{d}=f_{c} \frac{v}{c} \cos \theta
$$

The magnitude of Doppler shift is depended on 


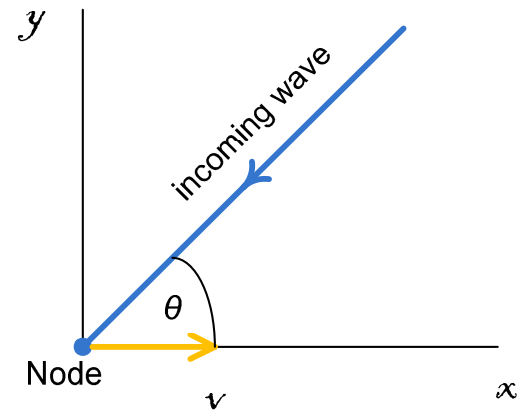

Fig. 4. A typical plane wave incident on a mobile receiver.

the angle formed by the terminal motion and the direction of radiation as shown in Figure 4. When the receiver move toward the transmitter and $\theta=$ $0^{\circ}$, the receiver feel the maximum positive Doppler shift. It is contrary when the receiver move away from the transmitter and $\Theta=180^{\circ}$. For multipath wave, the Doppler shift for path is given by:

$$
D_{i}=f_{c} \tau_{i}^{\prime}(t)
$$

Where $\tau_{i}^{\prime}(t)$ is the propagation delay at time $\mathrm{t}$ from the transmitter to the receiver on path $i$ which can be expressed as:

$$
\tau_{i}^{\prime}(t)=v_{i} / c
$$

Where $v_{i}$ is the velocity of th path.

When the different paths have different Doppler shifts, the magnitude of impulse response (attenuation) changes significantly at the time--scale inversely proportional to the largest difference between the Doppler shifts which is called the Doppler spread $\left(D_{\mathrm{s}}\right)$.

$$
\mathrm{D}_{s}:=\max _{i, j} f_{c}\left|\tau_{i}^{\prime}(t)-\tau_{j}^{\prime}(t)\right|
$$

The interval over which impulse response changes significantly as a function of time is define as the coherence time $\left(T_{c}\right)$ of a wireless channel [15]. The attenuations at two time instants separated by more than the coherence time are weakly correlated [16]. This coherence time is related to the Doppler frequency which is roughly expressed as:

$$
T_{c} \approx \frac{1}{D_{s}} \approx \frac{1}{f_{d}} \approx \frac{c}{v f_{c}}
$$

\section{THE PROPOSED METHOD}

Global System for Mobile (GSM) combines frequency division duplex (FDD) system and Time Division Multiple Access (TDMA) in such that $\mathrm{ev}^{-}$ ery band is divided into sub-channels and each sub-channel is shared by eight users in a time-division [14]. Therefore, this time-division fashion is adapted to the $\mathrm{CCN}$ to facilitate the movement of data source node. The adaptation is done by using buffer or queue module which is provided in OPNET [16]. Queue module in OPNET is able to access queuing facilities. It has interval time between scheduled self-interrupts where the incoming packet is pushed into a buffer and the self-interrupt will trigger the slot state for removing the packet from the buffer. This removal rate is fixed and act like a time-division access for every data transmission in the proposed method which is called the interval access time.

The environment which is used in the proposed method contains of three nodes: the client node, the access point node and the data source node. The client node contains two processor namely, the requester and the sink. The requester processor manages to produce the interest packets which are sent to the data source. The sink processor manages to receive the data packet and record the acceptance ratio by comparing the number of $\mathrm{re}^{-}$ ceived data packet with the number of interest packet sent. The data source contains one processor to produce data packet and reply the incoming interest packets which is called the replier. The access point connects the wireless media and the wire media.

The communication model of the proposed method is shown in Figure 5. There are four buffers used in the model. One buffer is in the client node to manage the transmission of the interest packet which has as the interval access time. A buffer in the data source node manages the transmission of the data packet which has as the inter- 


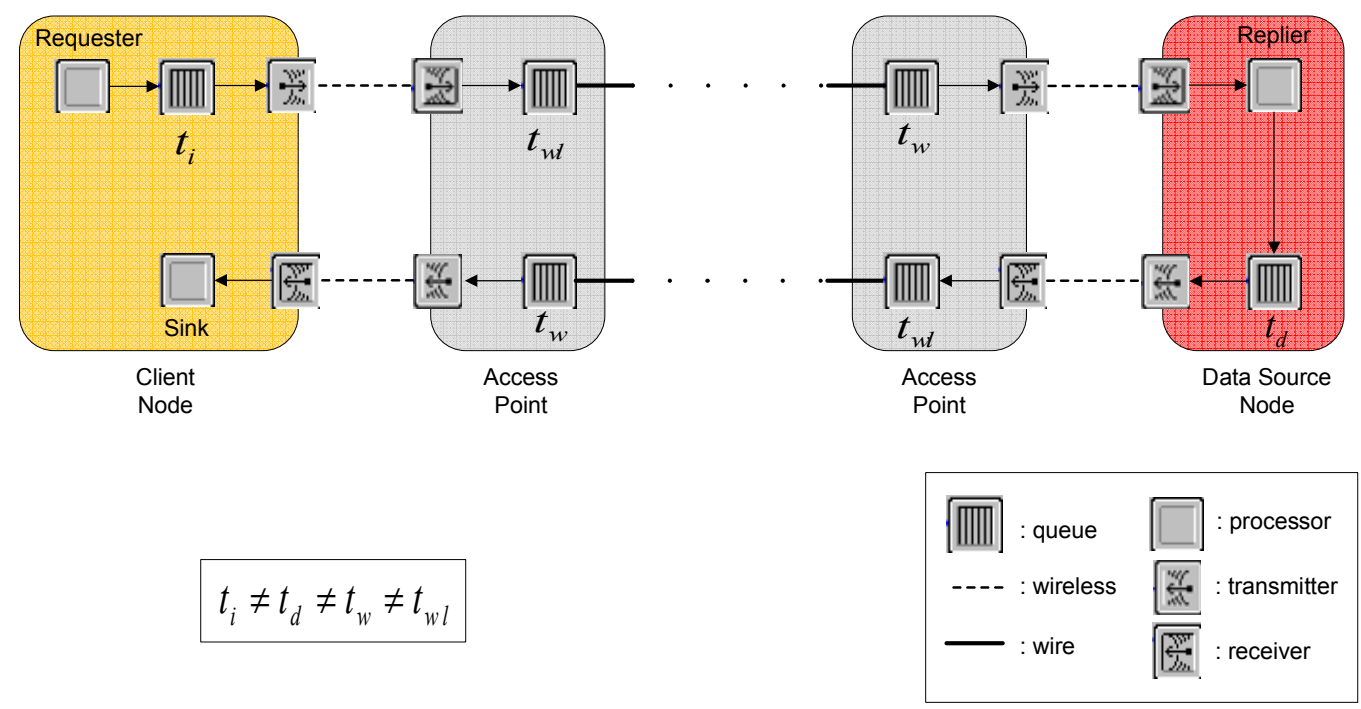

Fig. 5. Communication model of proposed method.

val access time. While two buffer in the access point node manage the incoming and outgoing packets from wireless to wire and vice versa. The buffer which manages the packets from wireless to wire have as the interval access time, while the other buffer which manages the packets from wire to wireless have as the interval access time. The value for $t_{i}, t_{d}, t_{\mathrm{w}}$ and are not the same.

\section{SIMULATION}

\subsection{Simulation Environment}

A simulation using OPNET is executed to test the proposed method and show the correlation of interval access time with the coherence time. Figure 8 shows the simulation environment which is used for testing the proposed method. There is a node called the client node which requests the data packets from the data source node. The client node and the data source node are at different domain which is connected by a router. The client node is at domain $\mathrm{A}$ which is connected to access point node of domain $\mathrm{A}\left(\mathrm{AP}_{\mathrm{A}}\right)$, while the data source node is at domain $\mathrm{B}$ which is connected to access point node of domain $\mathrm{B}\left(\mathrm{AP}_{\mathrm{B}}\right)$. While the client node stands still at the same position, The data source node moves from point I to point ii but still at the same domain.

There are two velocities of the data source nodes which are tested in this simulation: $50 \mathrm{~m} / \mathrm{s}$ and 25 $\mathrm{m} / \mathrm{s}$. The other parameters of simulation are shown in Table 1. The acceptance ratio of the client node is then tested with the varied of the interval access time and the velocity of the data source node.

Table 1. Simulation parameter

\begin{tabular}{|l|c|}
\hline Parameter & Value \\
\hline \hline$\Phi$ & $45^{\circ}$ \\
\hline $\mathrm{d}$ & $3 \mathrm{~km}$ \\
\hline & $30 \mathrm{MHz}$ \\
\hline Interest interval rate & $5 \mathrm{~s}$ \\
\hline Altitude & $30 \mathrm{~cm}$ \\
\hline
\end{tabular}

\subsection{Simulation Result}

The variation of $t_{i}, t_{d}, t_{\mathrm{w}}$ and is shown in Figure 7 and Figure 8 with their correlation with the acceptance ratio of the client node. The figures show that as the interval access time get bigger; the acceptance ratio of client node is become lower. The acceptance ratio is decrease significantly after the 


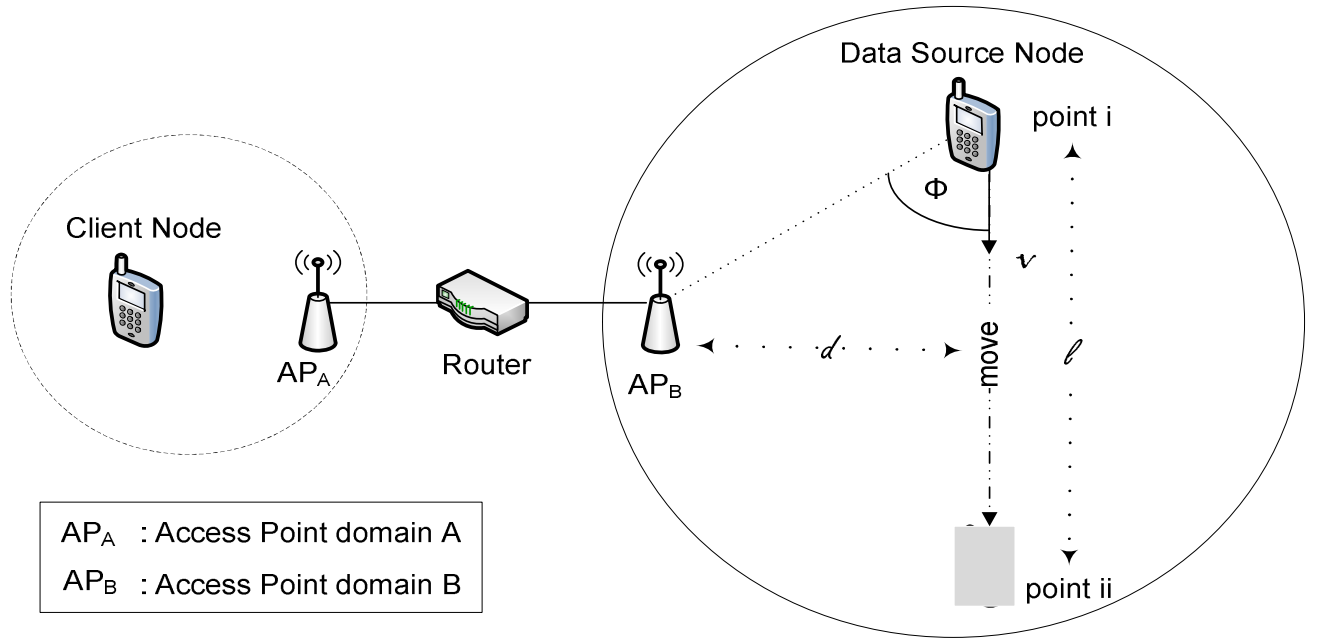

Fig. 6. Simulation environment.

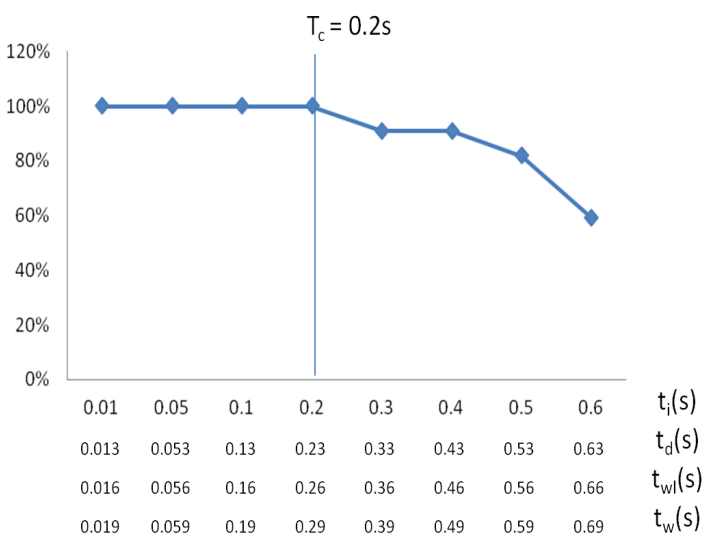

Fig. 7. Acceptance ratio of client node at $=50 \mathrm{~m} / \mathrm{s}$.

interval access time is bigger than the coherence time. It can be concluded from the result that the proposed method is worked as long as the interval access time is less than the coherence time.

The Doppler shift in this simulation change from $-D_{i}$ to $+D_{i}$. This rapid change in frequency, $d f_{d} /$ $d t$, is sometimes referred to as frequency slewing [14]. A good receiver must be capable of tracking the frequency slew rates.

\section{CONCLUSION}

The data source node movement triggers problem either when it moves to another domain or still

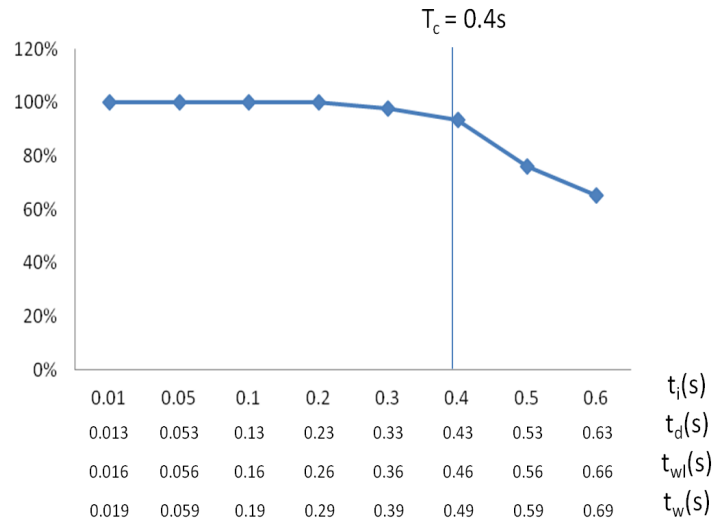

Fig. 8. Acceptance ratio of client node at $=25 \mathrm{~m} / \mathrm{s}$.

on the same domain. For the data source node movement at the same domain, fading and Doppler effect influence the packets transmission between the data source node and the access point node. This influence can be shown at the acceptance ratio of the client node. The simulation result shows that the time-division access method can defeat the side effect of intra-domain data source movement as long as the interval access time is less than the coherence time.

\section{REFERENCE}

[1] G. Tyson, N. Sastry, I. Rimac, R. Cuevas, and 
A. Mauthe, "A Survey of Mobility in Information-Centric Networks: Challenges and Research Directions," Proceeding of $A C M$ Workshop on Emerging Name-Oriented Mobile Networking Design, pp. 1-6, 2012.

[2] D. Kim, "Content Centric Networking Naming Scheme for Efficient Data Sharing", Journal of Korea Multimedia Society, Vol. 15, No. 9, pp. 1126-1132, 2012.

[3] A. Ghodsi, T. Koponen, B. Raghavan, S. Shenker, A. Singla, and J. Wilcox, "InformationCentric Networking: Seeing the Forest for the Trees," Proceeding of ACM SIGCOMM Workshop on Hot Topics in Networks, pp. 1-6, 2011.

[ 4 ] D. Kim, J. Kim, Y. Kim, H. Yoon, and I. Yeom, "Mobility Support in Content Centric Networks," Proceeding of Workshop on Information-centric Networking, pp. 13-18, 2012.

[5] O. Priyono, G.O. Yoon, and W. Hwang, "Early Mobility Notification in Content Centric Network using SIP," Proceeding of International Conference on Multimedia Information Technology and Applications, pp. 98-101, 2013.

[6] The Impact of mobility, https://www.cs.purdue.edu/homes/park/cs422-datalink_wireless-2-11f.pdf, 2014.

[7] A. Umar, Mobile Computing and Wireless Communications, NGE Solutions INC., Pennsylvania, 2004.

[ 8 ] J. Lee, S. Cho, and D. Kim, "Device Mobility Management in Content-Centric Network," IEEE Communication Magazine, Vol. 50, Issue 12, pp. 28-34, 2012.
[9] V. Jacobson, D.K. Smetters, J.D. Thornton, M. F. Plass, N.H. Briggs, and R.L. Braynard, "Networking Named Content," Proceeding of International Conference on Emerging Networking Experiments and Technologies, pp. 1-12, 2009.

[10] B. Ahlgren, C. Dannewitz, C. Imbrenda, D. Kutscher, and B. Ohlman, "A Survey of Information-Centric Networking," IEEE Communications Magazine, Vol. 50, Issue 7, pp. 2636, 2012.

[11] O. Priyono and W. Hwang, "The Impacts of Node Movement for Package Acceptance Ability of Client Node in Content-Centric Networking," Proceeding of KMMS Fall Conference, pp. 288, 2013.

[12] A. Paulus, E. Setijadi, and G. Hendrantoro, "Analisis Efek Doppler Pada Sistem Komunikasi ITS-SAT,” Journal Teknik Pomits, Vol. 2, No. 2, pp. 223-228, 2013.

[13] G.L. Stuber, Principles of Mobile Communication $2^{\text {nd }}$ edition, Kluwer Academic Publishers, Massachusetts, 2003.

[14] S. Haykin and M. Moher, Modern Wireless Communications, Pearson Prentice Hall, New Jersey, 2005.

[15] D. Tse and P. Viswanath, Fundamentals of Wireless Communication, Cambridge University Press, New York, 2005.

[16] A. Kumar, D. Manjunath, and J. Kuri, Wireless Networking, Morgan Kaufmann Publishers, Massachusetts, 2008.

[17] Z. Lu and H. Yang, Unlocking the Power of OPNET Modeler, Cambridge University Press, New York, 2012. 


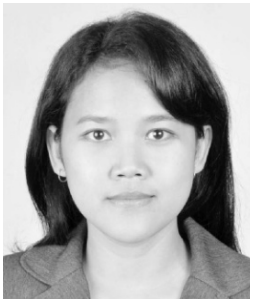

\section{Olivica Priyono}

is currently Master candidate at Inje University, Gyeongnam, Republic of Korea since September 2012. She received her B.S degree in Electrical Engineering sub major Telecommunication from Institut Teknologi Bandung, Bandung, Indonesia in 2008. Her research interest is mobility aspect in Content-Centric Networking.

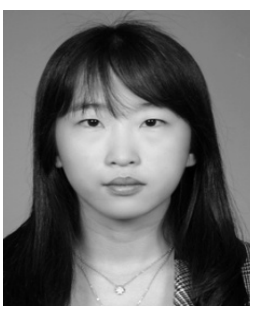

\section{In-Yeup Kong}

received her B.S. degree, M.S. degree and Ph.D Degree from Pusan National University, Pusan, Republic of Korea, in 2000, 2002, and 2007. Since September 2008, she has been an associate pro- $^{-}$ fessor at Kumoh National Institute of Technology, Gumi, Republic of Korea. Her research interests are in next-generation internet, and embedded systems. Mrs. Kong is a member of the IEICE.

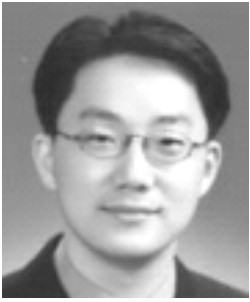

\section{Won-Joo Hwang}

received his Ph.D Degree in Information Systems Engineering from Osaka University Japan in 2002. He received his B.S. and M.S. degree in $\mathrm{Compu}^{-}$ ter Engineering from Pusan National University, Pusan, Republic of Korea, in 1998 and 2000. Since September 2002, he has been an associate professor at Inje University, Gyeongnam, Republic of Korea. His research interests are in network optimization and cross layer design. Mr. Hwang is a member of the IEICE and IEEE. 